\title{
Coccidioidomicosis extrapulmonar. Presentación de un caso y revisión de la literatura
}

\author{
Alejandro Avilés-Salas, Yolanda Quintero-Cuadra y Patricia Cornejo-Juárez
}

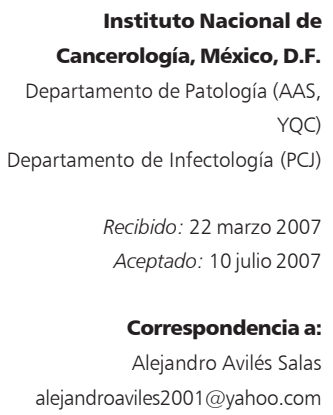

Instituto Nacional de Cancerología, México, D.F. YQC) C)
L.

a coccidioidomicosis o enfermedad de PosadasBuenos Aires, Argentina. Posteriormente, Rixford y Gilchrist, lograron transmitir en forma experimental la infección a animales de laboratorio ${ }^{1}$. Los agentes causales son hongos del género Coccidioides, los cuales incluyen la especie californiana $(C$. immitis $)$ y las especies recién nombradas no californianas $(C$. posadasii) ${ }^{2}$.

El área endémica principal de la coccidioidomicosis está localizada en las zonas áridas del sudoeste de los Estados Unidos, alcanzando su prevalencia máxima en el Valle de San Joaquín, California. En México abarca la región noroeste de Baja California, Sonora, Chihuahua y Sinaloa. En Centroamérica se han encontrado dos áreas endémicas; el Valle de Montagua en Guatemala y Comayagua en Honduras. En América del Sur, las principales áreas endémicas se localizan en el noreste de Venezuela, en las pampas abiertas del gran Chaco en Paraguay, en las zonas áridas de la Patagonia central en Argentina, en los departamentos de Guajira y Magdalena en Colombia; así como en los Estados de Piauí, Bahía, Ceará y Maranhao, al noreste de Brasil $^{1,3,4}$.

Las mujeres embarazadas, pacientes con procesos neoplásicos, sometidos a trasplantes de órganos y aquellos con síndrome de inmunodeficiencia adquirida son más susceptibles de enfermedad diseminada,

con afección de órganos como meninges, piel, hueso, articulaciones, mediastino y ganglios linfáticos ${ }^{5-7}$.

Paciente masculino, con 20 años de edad, soltero, heterosexual, campesino, con nivel socioeconómico bajo; sin antecedentes patológicos heredo-familiares ni personales de importancia, procedente de una comunidad rural del Estado de México. Señaló no haber realizado viajes a zonas endémicas de coccidioidomicosis dentro o fuera de la República Mexicana. Ingresó al Servicio de Hematología con el diagnóstico de probable linfoma no Hodgkin (LNH), refiriendo tumor de crecimiento rápido en la región cervical, con dos meses de evolución, acompañado de astenia, adinamia, cefalea, pérdida de peso y fiebre no cuantificada. En la exploración física se palparon múltiples adenopatías cervicales, la mayor de estas midió $3 \mathrm{~cm}$ de diámetro mayor y se localizó en el nivel IV del lado izquierdo. Se realizó biopsia de un ganglio cervical; sin embargo, no se envió muestra del tejido ganglionar a cultivo, ni se realizó estudio serológico, dada la consideración diagnóstica inicial de LNH. El estudio microscópico mostró 


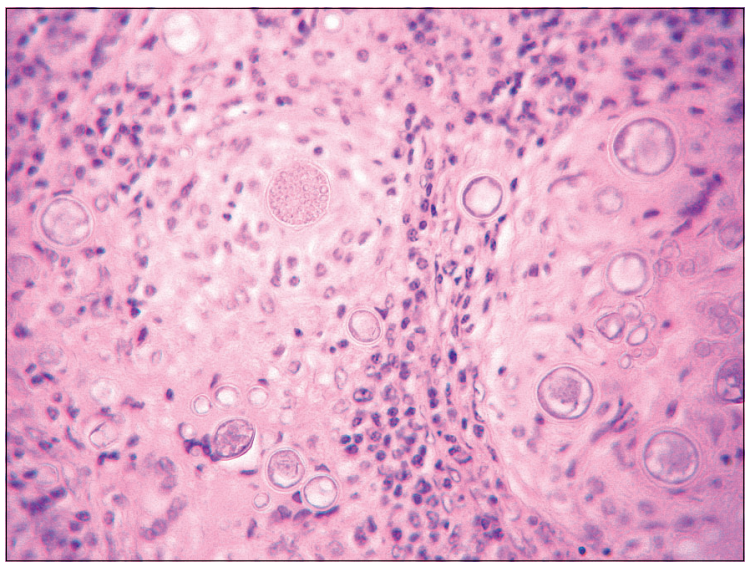

Figura 1. Corte histológico de ganglio linfático que muestra granulomas con presencia de esférulas (tinción de H-E, 400x).

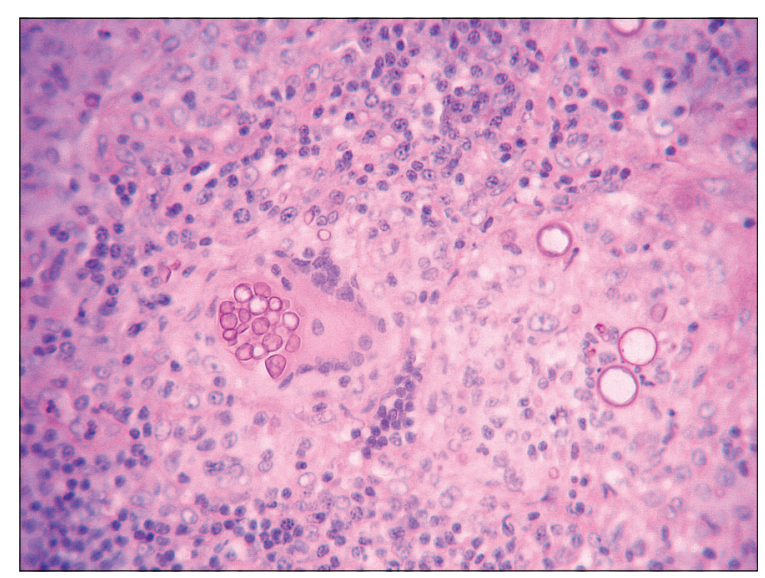

Figura 2. Célula gigante fagocitando esférula con múltiples endosporas en su interior (tinción de ácido periódico de Schiff, 400x).

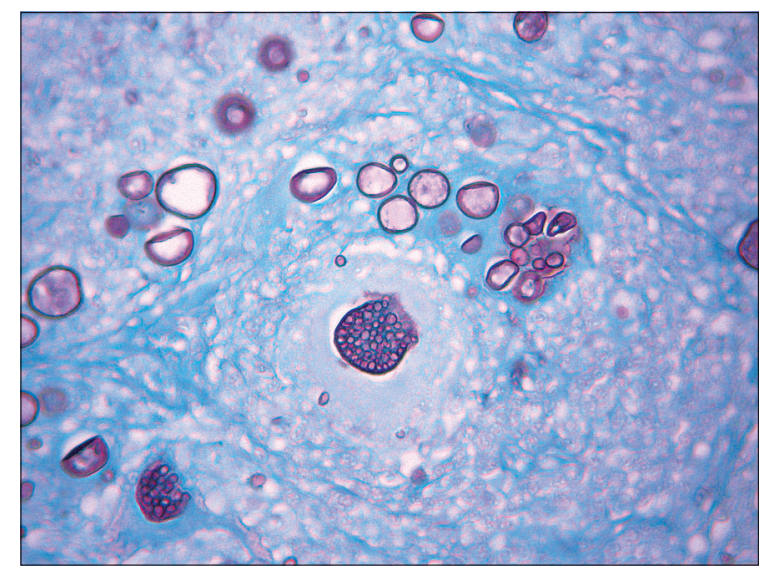

Figura 3. Endosporas y esférulas de pared gruesa (tinción de Grocott, 400x) tinción de Grocott se hicieron más evidentes tanto esférulas como endosporas (Figura 3), lo cual corroboró el diagnóstico de coccidioidomicosis. La prueba de ELISA para VIH fue negativa y se excluyeron otras causas de inmunosupresión. El paciente fue egresado y referido a su centro de salud.

\section{Discusión}

En E.U.A. ocurren, anualmente, más de 150 mil nuevos casos de coccidioidomicosis, de los cuales $60 \%$ son asintomáticos ${ }^{2}$. En México, la infección se ha informado en todos los grupos etarios; sin embargo, es más frecuente entre campesinos y trabajadores que emigran temporalmente a áreas endémicas; así como en arqueólogos, antropólogos y trabajadores de la construcción ${ }^{1}$.

Coccidioides immitis es un hongo dimórfico que se presenta como micelio o esférula. La fase micelial tiene su hábitat natural en los suelos de las regiones áridas y semiáridas de América. Las hifas tienen una morfología tubular y cuando maduran se fragmentan formando artroconidias con forma de barril, siendo la forma infectante del hongo. Estas esporas se desprenden al moverse la tierra seca, o bien, se desplazan con las corrientes de aire, hasta llegar a otro sitio en el suelo (ciclo saprobiótico); sin embargo, también pueden ser inhaladas por los animales o el hombre. El personal de laboratorio que manipula cultivos de C. immitis/posadasii debe tomar todas las medidas de bioseguridad, dada la contagiosidad de este microorganismo.

Dentro de los pulmones, el microorganismo induce una respuesta inflamatoria aguda y se desarrolla formando una esférula $(30-80 \mu \mathrm{m})$ de pared gruesa y birrefringente, que contiene en su interior endosporas (1-5 $\mu \mathrm{m})$. Una esférula madura puede contener hasta 800 endosporas y cuando se rompe, las endosporas quedan libres y cada una de ellas tiene la capacidad de formar una nueva esférula (ciclo parasitario-tisular). Cuando las esférulas presentes en los exudados o en los restos de tejidos de los animales muertos regresan al suelo, la endospora forma una yema alargada que se transforma en hifa, continuando así, un nuevo ciclo saprobiótico $^{1,8}$.

La infección, generalmente, se adquiere por inhalación de las artroconidias y produce un espectro amplio de manifestaciones clínico-patológicas. La infección 
primaria es asintomática en $\sim 60 \%$ de los casos. Las formas leves o moderadas representan $\sim 39 \%$, los síntomas aparecen en 7 a 21 días después de la exposición y se caracterizan por fiebre, tos, disnea, dolor torácico o cuadros gripales inespecíficos que curan espontáneamente, dando imágenes radiológicas de infiltrados neumónicos y adenopatías mediastínicas.

Diferentes manifestaciones cutáneas se han asociado con la coccidioidomicosis, siendo el eritema nodoso la lesión cutánea más informada. Aproximadamente $12 \%$ de los pacientes con enfermedad pulmonar sintomática desarrollan un exantema generalizado, el cual se hace presente durante los primeros días de la infección. Se debe considerar la sospecha clínica de coccidioidomicosis ante la aparición de un exantema cutáneo generalizado, fiebre, compromiso del estado general y síntomas respiratorios, en pacientes que viven o han viajado a áreas endémicas 9 .

Los casos diseminados representan $\sim 1 \%$, se encuentran con mayor frecuencia en pacientes africanos, norteamericanos, mexicanos y filipinos. Predominan en el género masculino, usualmente, en un contexto de inmunosupresión. Afectan comúnmente la piel, las meninges y huesos, así como sitios menos comunes como la glándula tiroides, la conjuntiva y el pericardio ${ }^{10-}$ ${ }^{12}$. Se han informado lesiones osteolíticas con reacción perióstica ${ }^{6}$, abscesos fríos, empiemas, fístulas tóracocostales, neumonías y formas miliares. La infección del tracto genital es una manifestación inusual de la coccidioidomicosis diseminada ${ }^{13,14}$

Son pocos los pacientes cuyo diagnóstico se ha realizado en biopsia de ganglio linfático ${ }^{6,15}$. En el presente caso, el diagnóstico se realizó en una biopsia de ganglio linfático, lo que representa una forma extrapulmonar o diseminada de la enfermedad. El paciente, por ser campesino, tuvo mayor riesgo de adquirir la infección, dado que este sector de la población está expuesto a la forma infectante del hongo (ciclo saprobiótico). Las formas diseminadas, usualmente, se presentan en un contexto de inmunosupresión, lo que no se corroboró en este paciente, siendo aparentemente sano. La impresión diagnóstica inicial fue LNH por ser un centro de referencia oncológica; sin embargo, se debieron tomar en cuenta otras posibilidades diagnósticas, incluyendo causas infecciosas.

Blair y cols, informaron 55 pacientes con neoplasias hematológicas y coccidioidomicosis, siendo el LNH y la leucemia linfocítica crónica las entidades más frecuentes. Las bases inmunológicas son poco claras; sin embargo, la alteración de la función de los linfocitos $\mathrm{T}$ juega un papel importante ${ }^{16,17}$. Se ha demostrado que los neutrófilos tienen dificultad para degradar la superficie anti-fagocítica de las esférulas. Los macrófagos juegan el papel más importante en la protección, pero sólo son efectivos si están presentes los linfocitos de ayuda (CD4+). Los pacientes con síndrome de inmunodeficiencia adquirida tienen gran riesgo de susceptibilidad, reactivación y diseminación, dada la disfunción de los linfocitos CD $4+{ }^{18}$.

El diagnóstico diferencial debe incluir tuberculosis, histoplasmosis, paracoccidioidomicosis, cromoblastomicosis, leishmaniasis y los micetomas.

El mejor método diagnóstico es el obtener una biopsia de tejido para estudio histopatológico y/o cultivo. La inmunidad natural de los humanos frente a C. immitis es muy elevada, puesto que $60 \%$ de los sujetos infectados permanecen asintomáticos y sólo 39\% tienen lesiones localizadas. La prueba de la coccidioidina consiste en la inyección intradérmica de coccidioidina micelial estandarizada. La reacción se lee a las 48 horas y se considera positiva cuando existe una zona de induración de $5 \mathrm{~mm}$ o más. La prueba de precipitación en tubo se hace positiva en las primeras semanas de la infección y se negativiza a los cuatro meses, con presencia de anticuerpos de la clase IgM. Esta prueba es muy específica y las reacciones cruzadas son excepcionales. La prueba de fijación del complemento aparece en el plazo de dos o tres meses en $90 \%$ de los pacientes sintomáticos $\mathrm{y}$, generalmente, se negativiza a los seis u ocho meses. Se recomienda efectuar una batería combinada de seropruebas seriadas, con el objeto de tener una interpretación más precisa de los resultados serológicos ${ }^{1}$.

Las guías de manejo de la coccidioidomicosis implica, inicialmente, corroborar la infección por C. immitis/ posadasii, definir la extensión de la enfermedad e identificar los factores del hospedero que acrecienten la gravedad de la enfermedad. A pesar de que la gran mayoría de los pacientes experimenta remisión de la enfermedad sin tratamiento antimicótico, se deben realizar controles médicos cada 3 a 6 meses por un período de dos años, con el objeto de documentar la resolución radiográfica, o identificar complicaciones pulmonares o extrapulmonares tan pronto como sea posible ${ }^{19}$.

El tratamiento de la coccidioidomicosis incluye anfotericina B deoxicolato (05-1,5 $\mathrm{mg} / \mathrm{kg} / \mathrm{día}$ iv), ketoconazol (400 mg/día vo), fluconazol (400-800 mg/ día iv o vo) e itraconazol (200 mg dos veces por día $)^{19,20}$. En algunos pacientes se ha usado la combinación de anfotericina B deoxicolato y un derivado azólico, especialmente cuando la infección es diseminada, o cuando hay progresión de la enfermedad durante el manejo con un solo agente. A pesar de que la combinación de varios fármacos puede mejorar la respuesta, no hay evidencia de que dicho abordaje sea superior al tratamiento con un solo fármaco ${ }^{19}$. Recientemente, han aparecido nuevos fármacos antimicóticos con posibles beneficios en el tratamiento de infecciones refracta- 
rias; dentro de éstos, caspofungina es efectiva en el manejo de la coccidioidomicosis murina, pero con gran variabilidad en la susceptibilidad in vitro. Voriconazol ha mostrado ser efectivo en ciertos pacientes; sin embargo, no ha sido aprobado por la FDA. Finalmente, posaconazol ha mostrado su efectividad in vitro e in vivo contra este hongo. Stevens y cols, publicaron un serie de 15 pacientes con coccidiodomicosis refractaria al tratamiento con anfotericina $\mathrm{B}$ deoxicolato \pm un azol, que fueron manejados con posaconazol $(800 \mathrm{mg} /$ día), informando una respuesta global de $73 \%$; por lo cual, este fármaco debe ser considerado como una opción importante en el tratamiento de pacientes con infecciones refractarias ${ }^{19,21}$.

En conclusión, se debe considerar el diagnóstico de coccidioidomicosis en aquellos pacientes con malestar general, fiebre, síntomas respiratorios y que vivan o hayan viajado a áreas endémicas. El diagnóstico se puede establecer mediante estudios serológicos y/o en una biopsia del tejido u órgano afectado, la cual se debe analizar desde el punto de vista microbiológico e histopatológico.

\section{Resumen}

La coccidioidomicosis es una micosis endémica en el sudoeste de los Estados Unidos, México y ciertas áreas de centro y Sudamérica. Los agentes causales son hongos del género Coccidioides. La infección primaria, habitualmente, involucra los pulmones, ocurriendo su diseminación en menos de $1 \%$ de los casos. $\mathrm{Si}$ bien la enfermedad extrapulmonar, usualmente, involucra la piel, el sistema nervioso central, los huesos y las articulaciones, tiene la capacidad de infectar cualquier órgano o tejido. Presentamos un caso de coccidioidomicosis extrapulmonar en un paciente masculino, con 20 años de edad, cuyo diagnóstico se estableció en una biopsia de ganglio linfático. La historia clínica no fue relevante y la biopsia se realizó debido a los hallazgos físicos sugerentes de linfoma.

\section{Referencias}

1.- Carrada-Bravo T. La coccidioidomicosis en los niños. Bol Med Hosp Infant Mex 1989; 46: $507-14$

2.- Fisher M C, Koenig G L, White T J, Taylor J W. Molecular and phenotypic description of Coccidioides posadasii $\mathrm{sp}$ nov previously recognized as the non-California population of Coccidioides immitis. Mycologia 2002; 94: 73-84.

3.- Wanke B, Lazera M, Monteiro P C, Lima F C, Leal M J, Ferreira Filho P L, et al. Investigation of an outbreak of endemic coccidioidomycosis in Brazil's northeastern state of Piaui with a review of the occurrence and distribution of Coccidioides immitis in three other Brazilian states. Mycopathologia 1999; 148: 57-67.

4.- Fisher M C, Koenig G L, White T J, San-Blas G, Negroni R, Alvarez I G, et al. Biogeographic range expansion into South America by Coccidiodes immitis mirrors New World patterns of human migration. Proc Natl Acad Sci USA 2001; 98: 4558-62.

5.- Stephany J D, Lucero S, Walsh A F. Mediastinal mass in a 27-year-old man. Arch Pathol Lab Med 2005; 129: 699-700.

6.- Smith M A, Anderson A E, Kostroff K. An unusual case of coccidioidomycosis. J Clin Microbiol 1994; 32: 1063-4.

7.- Crum-Cianflone N F, Truett A A,
Teneza-Mora N, Maves R C, Chun H M, Bavaro M F, et al. Unusual presentations of coccidioidomycosis. A case series and review of the literature. Medicine 2006: 85; 26377.

8.- Low W S, Seid A B, Pransky S M, Kearns D B. Coccidioides immitis subperiostal abscess of the temporal bone in a child. Arch Otolaryngol Head Neck Surg 1996; 122: 189-92.

9.- DiCaudo D J, Yiannias J A, Laman S D, Warschaw K E. The exanthem of acute pulmonary coccidioidomycosis. Clinical and histopathologic features of 3 cases and review of the literature. Arch Dermatol 2006; 142: 744-6.

10.- Sandoval J, Shank J, Morgan S, Agudelo J, Price C. Midfoot coccidioidal osteomyelitis. J Bone Joint Surg 2006; 88: 861-5.

11.- Smilack J, Argueta R. Coccidioidal infection of the thyroid. Arch Intern Med 1998; 158: 89-92.

12.- Galgiani J N. Coccidioidomycosis. West J Med 1993; 159: 153-71.

13.- Sohail M, Andrews P, Blair J. Coccidioidomycosis of the male genital tract. J Urol 2005; 173: 1978-82.

14.- Liao J C, Reiter R E. Coccidiodomycosis presenting as testicular mass. J Urol 2001; 166: 1396.

15.- Hernández J L, Echevarria S, García-Valtuille A, Mazorra F, Salesa R. Atypical coccidioidomycosis in an AIDS patient successfully treated with fluconazole. Eur J Clin Microbiol Infect Dis 1997; 16 : 592-4.

16.- Blair J E, Smilack J D, Caples S M. Coccidioidomycosis in patients with hematologic malignancies. Arch Intern Med 2005; 165: 113-17.

17.- Drutz D J, Hupper M. Coccidioidomycosis: factors affecting the host-parasite interaction. J Infect Dis 1983; 147: 372-90.

18.- Bronnimann D A, Adam R D, Galgiani J N, Habib M P, Petersen E A, Porter B, et al. Coccidioidomycosis in the acquired immunodeficiency syndrome. Ann Intern Med 1987; 106: 372-9.

19. - Galgiani J N, Ampel N M, Blair J E, Catanzaro A, Johnson R H, Stevens D A, et a. Infectious Diseases Society of America. Coccidioidomycosis. Clin Infect Dis 2005; 41: 1217-23.

20.- Galgiani J N, Catanzaro A, Cloud G A, Johnson R H, Williams P L, Mirels L F, et al. Comparison of oral fluconazole and itraconazole for progressive, nonmeningeal coccidioidomycosis. A randomized, doubleblind trial. Mycoses Study Group. Ann Intern Med 2000; 133: 676-86.

21.- Stevens D A, Rendon A, Gaona-Flores V, Catanzaro A, Anstead G M, Pedicone L, et al. Posaconazole therapy for chronic refractory coccidioidomicosis. Chest 2007; Jun 15: [Epub ahead of print]. 